\title{
Admission D-dimer testing for differentiating acute aortic dissection from other causes of acute chest pain
}

Wenlong Li ${ }^{1}$, Bi Huang ${ }^{2}$, Li Tian², Yanmin Yang ${ }^{2}$, Weili Zhang ${ }^{1}$, Xiaojian Wang ${ }^{1}$, Jingzhou Chen ${ }^{1}$, Kai Sun ${ }^{1}$, Rutai Hui ${ }^{1}$, Xiaohan Fan ${ }^{1}$

${ }^{1}$ State Key Laboratory of Cardiovascular Disease, Fuwai Hospital, Chinese Academy
of Medical Sciences \& Peking Union Medical College, Beijing, China
2Emergency and Critical Care Center of Cardiovascular Department, Fuwai Hospital,
Chinese Academy of Medical Sciences \& Peking Union Medical College, Beijing, China

Submitted: 14 November 2014

Accepted: 8 March 2015

Arch Med Sci 2017; 13, 3: 591-596

DOI: https://doi.org/10.5114/aoms.2017.67280

Copyright $\odot 2016$ Termedia \& Banach

\section{Abstract}

Introduction: The present study aims to evaluate the utility of D-dimer testing for differentiating the causes of acute chest pain, including acute aortic dissection (AAD), pulmonary embolism (PE), acute myocardial infarction (AMI), unstable angina (UA), and other uncertain diagnoses of chest pain.

Material and methods: Consecutive patients admitted for acute chest pain within $24 \mathrm{~h}$ from symptom onset were enrolled prospectively, and plasma D-dimer levels were measured on admission. Diagnoses of AAD, PE, AMI, and UA were confirmed by standard methods.

Results: A total of 790 patients were enrolled, including 202 AAD, 43 PE, $315 \mathrm{AMI}, 136 \mathrm{UA}$, and 94 cases of other uncertain diagnoses. D-dimer levels were significantly higher in patients with $A A D$ and $P E$ than in those with AMI, UA, and other uncertain diagnoses $(p<0.001)$, but they were comparable between patients with AAD and PE $(p=0.065)$. Moreover, patients with type A AAD had higher D-dimer levels than those with type $B A A D$ $(p=0.022)$. Receiver operating characteristic (ROC) curve analysis showed that a D-dimer level $<0.5 \mu \mathrm{g} / \mathrm{ml}$ was a good predictor for ruling out AAD, with a sensitivity of $94.0 \%$ and a specificity of $56.8 \%$. At a cut-off level of $0.5 \mu \mathrm{g} / \mathrm{ml}$, the negative and positive likelihood ratios were 0.10 and 2.18 , respectively, with a positive predictive value of $42.6 \%$ and a negative predictive value of $96.6 \%$.

Conclusions: The D-dimer level within $24 \mathrm{~h}$ after symptom onset might be helpful for differentiating AAD from other causes of chest pain.

Key words: pulmonary embolism, acute myocardial infarction, unstable angina, chest pain.

\section{Introduction}

Acute aortic dissection (AAD) is a relatively uncommon medical emergency with a high mortality after symptom onset. The mortality of acute type $A$ aortic dissection increases by $1-2 \%$ per hour during the first $48 \mathrm{~h}$ if no treatment is received [1]. Meanwhile, other common causes of acute chest pain, such as acute myocardial infarction (AMI) and pulmonary embolism (PE), also require rapid differentiation from AAD due to their critical and lethal characteristics [2]. However, the misdiagnosis rate of AAD has been reported to be approximately $30 \%$ on initial evalu-

\author{
Corresponding authors: \\ Xiaohan Fan, Rutai Hui \\ State Key Laboratory \\ of Cardiovascular Disease \\ Fuwai Hospital \\ Chinese Academy \\ of Medical Sciences \\ \& Peking Union \\ Medical College \\ 167 Beilishi Road \\ Beijing 100037, China \\ Phone: +86 10-88398154, \\ $+8610-88398154$ \\ Fax: +86 10-68331730, \\ +86 10-68331730 \\ E-mail: fanxiaohan@ \\ fuwaihospital.org
}


ation $[3,4]$. Currently, noninvasive imaging modalities, including enhanced computed tomography (CT), transesophageal echocardiography (TEE) and magnetic resonance imaging (MRI), have been developed to improve the diagnosis of $A A D$, but these imaging modalities are expensive, time-consuming and unavailable at the bedside. Therefore, a rapid, cheap, reliable and sensitive laboratory test is urgently needed to diagnose AAD.

D-dimer, the degradation product of cross linked fibrin, is significantly elevated in AAD patients [5-8] and has been suggested for use as a complementary marker to rule out AAD [5-7, 9-11]. However, in real-world clinical practice, $A A D, P E$ and $A M I$ are all thrombogenic diseases with high mortality, and whether the D-dimer level is helpful for differentiating these diseases remains to be elucidated. We therefore conducted a prospective cohort study to evaluate the validity and reliability of D-dimer level for differentiating $A A D$ from other types of acute chest pain, including $P E, A M I$, unstable angina (UA), and other uncertain diagnoses of chest pain.

\section{Material and methods}

\section{Study population}

A single-center, prospective cohort study was conducted in Fuwai Hospital (the National Center for Cardiovascular Diseases in China) from January 2009 to January 2010. A series of consecutive patients with acute chest pain who presented to the emergency department (ED) of Fuwai Hospital within $24 \mathrm{~h}$ of symptom onset were enrolled in a prospective manner. Baseline clinical characteristics such as sex, age, Stanford types of AAD, intervals from onset of symptoms to hospital admission, medical histories, baseline parameters of physical examinations and laboratory tests including C-reactive protein (CRP), imaging examinations, in-hospital managements, ED diagnosis and discharge diagnosis were recorded according to pre-designed case report forms. The study protocols were approved by the appropriate institutional review boards of Fuwai Hospital and complied with the Declaration of Helsinki. All subjects provided written informed consent.

\section{D-dimer test and diagnosis}

Plasma D-dimer levels were measured using a stago-evolution device (France) in patients with chest pain immediately following admission. The results collected are expressed in micrograms per milliliter. The effective detection range of the assay is $0.22-20 \mu \mathrm{g} / \mathrm{ml}$. Diagnoses of AAD and PE were confirmed by aorta or pulmonary angiography with multi-detector CT scan. Acute myocardial infarction was confirmed by acute chest pain, ele- vated cardiac-enzyme levels (cardiac troponin I or $\mathrm{T}$, or the MB fraction of creatine kinase exceeded the $99^{\text {th }}$ percentile upper reference limit), documented findings of a new ST segment elevation/ depression or a new $T$ wave inversion on electrocardiography, and/or with evidence of obstructive coronary artery on angiography. Unstable angina was confirmed by chest pain, ST segment depression or T wave changes with evidence of obstructive coronary artery on angiography, but without the elevation of cardiac enzymes.

\section{Statistical analysis}

Continuous variables are presented as mean \pm SD or median and interquartile range according to whether they follow Gaussian distributions. Categorical data are presented as numbers and proportions. Baseline characteristics between groups were compared using Student's $t$ test or the nonparametric Mann-Whitney test for continuous data and the $\chi^{2}$ test for categorical data. Receiver-operating characteristic (ROC) curves were constructed to calculate the sensitivity for AAD. The area under the curve (AUC) was calculated. A p-value $<0.05$ was considered statistically significant. The statistical calculations were performed with SPSS 19.0 (SPSS Inc., Chicago, Illinois, USA).

\section{Results}

A total of 790 patients were enrolled, including $202 \mathrm{AAD}, 43 \mathrm{PE}, 315 \mathrm{AMI}, 136 \mathrm{UA}$, and 94 cases with other uncertain diagnoses. Of the 202 AAD patients confirmed by CT angiography, 119 (58.9\%) were Stanford type A AAD cases and 83 (41.0\%) were Stanford type B AAD cases.

Patient demographics and baseline characteristics are shown in Table I. Compared to the patients with other causes of chest pain, AAD patients were more likely to be younger and male and tended to have concomitant hypertension but rarely have diabetes mellitus (all $p<0.001$ ).

The D-dimer level was elevated $(>0.50 \mu \mathrm{g} / \mathrm{ml})$ in 190 (94.1\%) AAD patients. The D-dimer level in AAD patients was approximately 9-fold higher than that in non-AAD patients (median: 4.19 vs. $0.45 \mu \mathrm{g} / \mathrm{ml}, p<0.05$ ). Figure 1 shows the $\mathrm{D}$-dimer level in patients with different causes of chest pain. The D-dimer level was significantly higher in patients with $A A D$ than in patients with UA (median: $0.38 \mu \mathrm{g} / \mathrm{ml}, p<0.001$ ), AMI (median: 0.45 $\mu \mathrm{g} / \mathrm{ml}, p<0.001)$ and other uncertain diagnoses (median: $0.44 \mu \mathrm{g} / \mathrm{ml}, p<0.001$ ), but it was comparable with that of PE patients (median: $2.72 \mu \mathrm{g}$ / $\mathrm{ml}, p=0.065$ ). Similarly, the D-dimer level in PE patients was significantly higher than that in patients with UA, AMI, or other uncertain diagnoses (all $p<0.001$ ). Moreover, patients with type A AAD 
Table I. Baseline characteristics of AAD patients and non-AAD (PE, UA, AMI, and uncertain diagnosis)

\begin{tabular}{|c|c|c|c|c|c|c|}
\hline \multirow[t]{2}{*}{ Parameter } & \multirow{2}{*}{$\begin{array}{c}\text { AAD } \\
(n=202)\end{array}$} & \multicolumn{4}{|c|}{ Non-AAD } & \multirow[t]{2}{*}{$P$-value } \\
\hline & & $\begin{array}{c}\text { PE } \\
(n=43)\end{array}$ & $\begin{array}{c}\text { UA } \\
(n=136)\end{array}$ & $\begin{array}{c}\text { AMI } \\
(n=315)\end{array}$ & $\begin{array}{c}\text { Other } \\
(n=94)\end{array}$ & \\
\hline Age [years] & $51 \pm 12$ & $55 \pm 17$ & $61 \pm 12$ & $60 \pm 12$ & $54 \pm 17$ & $<0.001$ \\
\hline Male, $n(\%)$ & $169(83.7)$ & $21(48.8)$ & $102(75.0)$ & $254(80.6)$ & $65(69.1)$ & $<0.001$ \\
\hline Systolic blood pressure [mm Hg] & $141 \pm 31$ & $129 \pm 21$ & $138 \pm 23$ & $128 \pm 23$ & $133 \pm 23$ & $<0.001$ \\
\hline Diastolic blood pressure [mm Hg] & $80 \pm 21$ & $81 \pm 10$ & $87 \pm 57$ & $79 \pm 14$ & $81 \pm 14$ & 0.535 \\
\hline Heart rate [beats per minute] & $81 \pm 19$ & $87 \pm 17$ & $72 \pm 13$ & $76 \pm 18$ & $80 \pm 28$ & $<0.001$ \\
\hline Body mass index $\left[\mathrm{kg} / \mathrm{m}^{2}\right]$ & $24.6 \pm 3.2$ & $25.7 \pm 3.7$ & $26.7 \pm 4.2$ & $25.5 \pm 3.4$ & $26.2 \pm 4.9$ & 0.450 \\
\hline Creatinine kinases [U/I] & $269 \pm 544$ & $85 \pm 61$ & $97 \pm 84$ & $497 \pm 688$ & $109 \pm 105$ & $<0.001$ \\
\hline Fasting blood glucose $[\mathrm{mmol} / \mathrm{l}]$ & $7.5 \pm 1.9$ & $6.3 \pm 1.6$ & $7.4 \pm 3.1$ & $8.4 \pm 3.4$ & $7.1 \pm 2.7$ & $<0.001$ \\
\hline Hypertension, $n$ (\%) & $133(65.8)$ & $13(31.0)$ & $86(63.2)$ & $161(51.3)$ & $42(46.2)$ & $<0.001$ \\
\hline Diabetes mellitus, $n$ (\%) & $5(2.5)$ & $2(4.8)$ & $31(22.8)$ & $68(21.7)$ & $13(14.3)$ & $<0.001$ \\
\hline Hypercholesterolemia, $n$ (\%) & $18(8.9)$ & $3(7.1)$ & $34(25.0)$ & $75(24.0)$ & $13(14.3)$ & $<0.001$ \\
\hline Stroke, $n(\%)$ & $10(5.0)$ & $2(4.8)$ & $13(9.6)$ & $33(10.5)$ & $7(7.7)$ & 0.471 \\
\hline Smoker, n (\%) & $64(31.7)$ & $7(16.7)$ & $31(22.8)$ & $105(33.5)$ & $18(19.8)$ & 0.060 \\
\hline Drinker, $n$ (\%) & $21(10.4)$ & $0(0.0)$ & $6(4.4)$ & $14(4.5)$ & $6(6.6)$ & 0.110 \\
\hline
\end{tabular}

$A A D$ - acute aortic dissection, $P E$ - pulmonary embolism, UA - unstable angina, AMI - acute myocardial infarction.

had higher D-dimer levels than those with type $B$ AAD (median: 4.64 vs. $4.0 \mu \mathrm{g} / \mathrm{ml}, p=0.022$ ).

Figure 2 shows the ROC for patients with $A A D$ versus non-AAD patients. The AUC value was 0.90 (95\% Cl: 0.87-0.93) for patients with AAD vs. all non-AAD patients. The AUC value was 0.59 (95\% Cl: 0.5-0.68) vs. PE, 0.91 (95\% Cl: 0.88-0.94) vs. AMI, 0.95 ( $95 \% \mathrm{Cl}: 0.93-0.97)$ vs. UA, and 0.93 (95\% Cl: 0.91-0.96) vs. patients with other uncertain diagnoses. Moreover, the best cut-off value of D-dimer for predicting PE was $1.14 \mu \mathrm{g} / \mathrm{ml}$ by ROC analysis with an AUC of 0.79 (95\% Cl: 0.74-0.84).

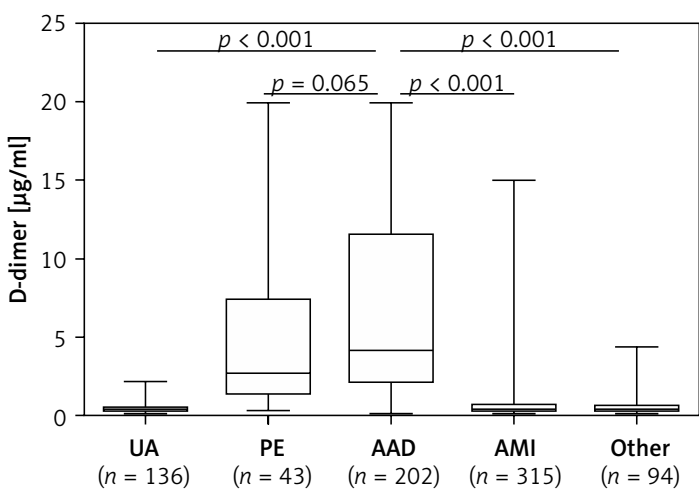

Figure 1. Comparison of D-dimer levels in patients admitted for chest pain

$A A D$ - acute aortic dissection, PE - pulmonary embolism, $U A$ - unstable angina, $A M I$ - acute myocardial infarction.
The sensitivity and specificity were $88.4 \%$ and $71.2 \%$, respectively.

The diagnostic performance at the cutoff level of $0.5 \mu \mathrm{g} / \mathrm{ml}$ was analyzed. At this cutoff level, the sensitivity was $94.0 \%$ and the specificity was $56.8 \%$ for AAD compared to non-AAD patients; the negative and positive likelihood ratio were 0.10 and 2.18 , respectively with a positive predictive value of $42.6 \%$ and a negative predictive value of $96.6 \%$. The specificity was $4 \%$ for PE, $56 \%$ for AMI, $72.9 \%$ for UA, and $65.1 \%$ for uncertain diagnostic cases (Table II).

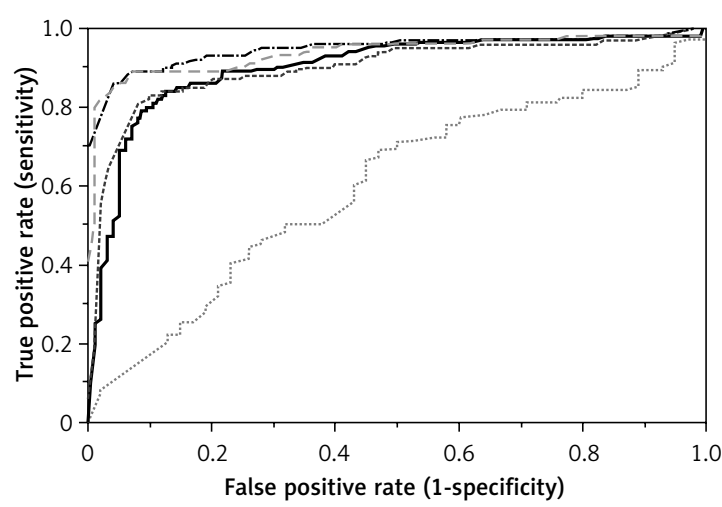

- $A A D$ vs. non-AAD -..-- AAD vs. AMI -.-.. AAD vs. UA

$$
\text { ....... AAD vs. PE - - AAD vs. other }
$$

Figure 2. ROC for the prediction by D-dimer level in patients with $A A D$ versus non-AAD

$A A D$ - acute aortic dissection, $P E$ - pulmonary embolism, $U A$ - unstable angina, $A M I$ - acute myocardial infarction. 
Table II. Diagnostic performance of D-dimer at the cutoff level of $0.5 \mu \mathrm{g} / \mathrm{ml}$

\begin{tabular}{|c|c|c|c|c|c|c|c|}
\hline Variable & Sensitivity (\%) & Specificity (\%) & Youden's index & PPV (\%) & NPV (\%) & PLR & NLR \\
\hline$A A D$ & 94.0 & & & & & & \\
\hline Non-AAD: & & 56.8 & 0.51 & 42.6 & 96.6 & 2.18 & 0.10 \\
\hline PE & & 4.0 & -0.02 & 81.1 & 14.2 & 0.97 & 1.25 \\
\hline AMI & & 56.0 & 0.49 & 57.5 & 93.5 & 2.11 & 0.12 \\
\hline UA & & 72.9 & 0.67 & 83.7 & 89.2 & 3.48 & 0.08 \\
\hline Uncertain & & 65.1 & 0.56 & 86.3 & 83.3 & 1.44 & 0.09 \\
\hline
\end{tabular}

\section{Discussion}

The present study demonstrated a significantly higher admission D-dimer level in patients with AAD within $24 \mathrm{~h}$ after symptom onset than those with AMI, UA, and other uncertain diagnoses. At the widely used cutoff level of $0.5 \mu \mathrm{g} / \mathrm{ml}$, a favorable negative likelihood ratio of 0.10 and negative predictive value of $96.6 \%$ were found in patients with AAD. However, the D-dimer level was not significantly different between patients with $A A D$ and PE. Our study suggests that a plasma D-dimer test within $24 \mathrm{~h}$ of symptom onset may be helpful for differentiating AAD and PE from other causes of acute chest pain.

Acute aortic dissection is a catastrophic medical emergency, which requires early and accurate diagnosis and treatment. Imaging modalities, in cluding enhanced CT and MRI, can facilitate an accurate diagnosis. However, these methods are limited due to unavailability at the bedside and their time-consuming nature, and they are not cost effective for routine screening. Thus, a rapid and reliable biomarker is urgently needed. Previous studies have evaluated several biomarkers for AAD, such as the smooth muscle myosin heavy chain [12-14], the BB-isozyme of creatine kinase [15], and calponin [16]. However, none of these markers have been adopted into routine clinical practice due to their inability to meet the requirements of a 'gold standard' biomarker including having adequate sensitivity and specificity in addition to a favorable time course of release that covers a time window necessary for nonambiguity in the clinical setting [17]. D-dimer is a fibrin fragment seen in coagulopathic disorders, and measurements are routinely used for the exclusion of venous thromboembolic diseases and PE [18-20]. In recent years, multiple studies have confirmed that $D$-dimer is elevated in AAD, and several studies have assessed its diagnostic value for AAD. However, at a defined cutoff value, the sensitivity and specificity of D-dimer for the diagnosis of AAD have been reported to vary, possibly due to differ- ent assay methods used in different studies. Generally, when a cutoff value of $0.5 \mu \mathrm{g} / \mathrm{ml}$ is used, the sensitivity and negative predictive value can reach almost $100 \%$ with a specificity of $54-68.6 \%$ $[5,9]$, and the specificity can be increased to $73 \%$ when the cutoff value is $0.626 \mu \mathrm{g} / \mathrm{ml}$ [6]. Shimony et al. [21] recently performed a meta-analysis of D-dimer to diagnose AAD and found that at a cutoff value of $0.5 \mu \mathrm{g} / \mathrm{ml}$, the sensitivity and negative predictive value were 0.97 and 0.96 , respectively. However, the specificity and positive predictive value were low, 0.56 and 0.60 , respectively. Moreover, the negative likelihood ratio showed an excellent discriminative ability (0.06), whereas the positive likelihood ratio did not (2.43). They concluded that a plasma D-dimer level $<0.5 \mu \mathrm{g} / \mathrm{ml}$ was a useful screening tool to identify patients who do not have AAD. Therefore, the plasma D-dimer level may thus be used to identify subjects who are unlikely to benefit from further aortic imaging. Our results were consistent with this study, suggesting that the cutoff value of D-dimer $<0.5 \mu \mathrm{g} /$ $\mathrm{ml}$, which is widely used for excluding PE [22], is also applicable for the exclusion of AAD. However, the D-dimer level in patients with $A A D$ is not always elevated, and several studies [23, 24], including ours, have observed this phenomenon. Hazui et al. [25] proposed that younger patients with a short dissection length and a thrombosed false lumen without ulcer-like projections may have false-negative D-dimer results. Therefore, patients who present classic characteristics of AAD but have a negative D-dimer test should receive further aortic imaging.

Due to its non-specific characteristics, an elevated D-dimer level is also seen in patients with other morbidities such as PE, AMI, UA, and other diseases. Therefore, further investigation is necessary to clarify whether D-dimer tests can differentiate $A A D$ from other diseases that presented with elevated D-dimer levels. Suzuki et al. [26] reported that when the cutoff level was $1.6 \mu \mathrm{g} / \mathrm{ml}$, D-dimer was a useful tool for differentiating $A A D$ from AMI, angina or other ischemic heart diseases 
within the first $6 \mathrm{~h}$, and when the cutoff value was 0.8 or $0.9 \mu \mathrm{g} / \mathrm{ml}$, the $\mathrm{D}$-dimer level could differentiate AAD from AMI [27]. Sakamoto et al. [28] also found that a cutoff value of $0.5 \mu \mathrm{g} / \mathrm{ml}$ was effective for distinguishing $A A D$ and PE from $A M I$, with a sensitivity of $68 \%$ and a specificity of $90 \%$. Although their results were mostly consistent with ours, the cutoff values used in these studies were different and the obtained D-dimer levels in various causes of acute chest pain varied greatly. One possible explanation for this variation was the different measurement equipment and the test strip used. Therefore, a standard and unified detection protocol may improve the heterogeneity of measurement, making the detection value more reliable.

Additionally, the D-dimer level was elevated in both $A A D$ and PE patients, with no significant difference in our study, consistent with the findings of Sakamoto et al. [28] and Eggebrecht et al. [6]. Given the high mortality of the two morbidities, immediate contrast CT imaging or tissue Doppler imaging [29] may be good choices to differentiate AAD from $P E$.

In the setting of AMI/UA, rupture of atherosclerotic plaques causes thrombopoiesis and activates fibrin degradation, leading to D-dimer formation. Therefore, D-dimer is elevated in patients with AMI/UA but not in patients with stable angina and healthy controls [30, 31]. Although the D-dimer level does not directly reflect the degree of myocardial damage, it has been confirmed that an elevated D-dimer level is a strong predictor of mortality in patients with AMI/UA $[32,33]$. Therefore, the D-dimer level is not only a useful tool for the differentiation of diagnoses, but it also plays an important role in the prognostic evaluation for some cardiovascular diseases.

Some limitations of the present study need to be addressed. First, although our study shows good prediction for AAD with the D-dimer level at the cutoff of $0.5 \mu \mathrm{g} / \mathrm{ml}$, the specificity is low (56.8\%). Indeed, D-dimer as a diagnostic biomarker of AAD did have some limitations due to the relatively high false positive rate. Therefore, for patients with a D-dimer level $>0.5 \mu \mathrm{g} / \mathrm{ml}$, the D-dimer level should be combined with other diagnostic tests, especially imaging tests, for an accurate diagnosis of AAD. Second, the small sample size of PE patients may affect the statistical power. Furthermore, the difference in D-dimer levels was not evaluated between patients with ST-segment elevation AMI and non-ST-segment elevation AMI. Therefore, further large, prospective, multi-center studies are needed.

In conclusion, the D-dimer level within $24 \mathrm{~h}$ after symptom onset might be helpful for differentiating patients with suspected AAD from other causes of chest pain.

\section{Acknowledgments}

The first two authors contributed equally to this study.

We wish to thank the patients for their participations in our study, and we are also grateful to other clinical doctors and nurses for their help in the study. This work was supported by a grant (81170286) from the National Natural Science Foundation of China to Dr. Fan Xiaohan.

\section{Conflict of interest}

The authors declare no conflict of interest.

\section{References}

1. Hirst AJ, Johns VJ, Kime SJ. Dissecting aneurysm of the aorta: a review of 505 cases. Medicine (Baltimore) 1958; 37: 217-79.

2. Erbel R, Alfonso F, Boileau C, et al. Diagnosis and management of aortic dissection. Eur Heart J 2001; 22: 1642-81.

3. von Kodolitsch Y, Nienaber CA, Dieckmann C, et al. Chest radiography for the diagnosis of acute aortic syndrome. Am J Med 2004; 116: 73-7.

4. Spittell PC, Spittell JJ, Joyce JW, et al. Clinical features and differential diagnosis of aortic dissection: experience with 236 cases (1980 through 1990). Mayo Clin Proc 1993; 68: 642-51.

5. Weber T, Hogler S, Auer J, et al. D-dimer in acute aortic dissection. Chest 2003; 123: 1375-8.

6. Eggebrecht $\mathrm{H}, \mathrm{Naber} \mathrm{CK}$, Bruch C, et al. Value of plasma fibrin D-dimers for detection of acute aortic dissection. J Am Coll Cardiol 2004; 44: 804-9.

7. Perez A, Abbet P, Drescher MJ. D-dimers in the emergency department evaluation of aortic dissection. Acad Emerg Med 2004; 11: 397-400.

8. Tian L, Fan X, Zhu J, Liang Y, Li J, Yang Y. Plasma D-dimer and in-hospital mortality in patients with Stanford type $A$ acute aortic dissection. Blood Coagul Fibrinolysis 2014; 25: 161-6.

9. Akutsu K, Sato N, Yamamoto T, et al. A rapid bedside D-dimer assay (cardiac D-dimer) for screening of clinically suspected acute aortic dissection. Circ J 2005; 69: 397-403.

10. Ohlmann P, Faure A, Morel O, et al. Diagnostic and prognostic value of circulating D-dimers in patients with acute aortic dissection. Crit Care Med 2006; 34: 1358-64.

11. Sbarouni E, Georgiadou P, Marathias A, Geroulanos S, Kremastinos DT. D-dimer and BNP levels in acute aortic dissection. Int J Cardiol 2007; 122: 170-2.

12. Suzuki T, Katoh $\mathrm{H}$, Watanabe $\mathrm{M}$, et al. Novel biochemical diagnostic method for aortic dissection. Results of a prospective study using an immunoassay of smooth muscle myosin heavy chain. Circulation 1996; 93: 1244-9.

13. Katoh H, Suzuki T, Hiroi Y, et al. Diagnosis of aortic dissection by immunoassay for circulating smooth muscle myosin. Lancet 1995; 345: 191-2.

14. Suzuki T, Katoh H, Tsuchio Y, et al. Diagnostic implications of elevated levels of smooth-muscle myosin heavy-chain protein in acute aortic dissection. The smooth muscle myosin heavy chain study. Ann Intern Med 2000; 133: 537-41.

15. Suzuki T, Katoh H, Kurabayashi M, Yazaki Y, Nagai R. Biochemical diagnosis of aortic dissection by raised con- 
centrations of creatine kinase BB-isozyme. Lancet 1997 ; 350: 784-5.

16. Suzuki T, Distante A, Zizza A, et al. Preliminary experience with the smooth muscle troponin-like protein, calponin, as a novel biomarker for diagnosing acute aortic dissection. Eur Heart J 2008; 29: 1439-45.

17. Suzuki T, Distante A, Eagle K. Biomarker-assisted diagnosis of acute aortic dissection: how far we have come and what to expect. Curr Opin Cardiol 2010; 25: 541-5.

18. Frost SD, Brotman DJ, Michota FA. Rational use of D-dimer measurement to exclude acute venous thromboembolic disease. Mayo Clin Proc 2003; 78: 1385-91.

19. Righini M, Van Es J, Den Exter PL, et al. Age-adjusted D-dimer cutoff levels to rule out pulmonary embolism: the ADJUST-PE study. JAMA 2014; 311: 1117-24.

20. Duru S, Kelesoglu A, Ardic S. Clinical update on pulmonary embolism. Arch Med Sci 2014; 10: 557-65.

21. Shimony A, Filion KB, Mottillo S, Dourian T, Eisenberg MJ. Meta-analysis of usefulness of d-dimer to diagnose acute aortic dissection. Am J Cardiol 2011; 107: 1227-34.

22. Eng CW, Wansaicheong G, Goh SK, Earnest A, Sum C. Exclusion of acute pulmonary embolism: computed tomography pulmonary angiogram or D-dimer? Singapore Med J 2009; 50: 403-6.

23. Paparella D, Malvindi PG, Scrascia G, et al. D-dimers are not always elevated in patients with acute aortic dissection. J Cardiovasc Med (Hagerstown) 2009; 10: 212-4.

24. Wiegand J, Koller M, Bingisser R. Does a negative D-dimer test rule out aortic dissection? Swiss Med Wkly 2007; 137: 462

25. Hazui $H$, Nishimoto $M$, Hoshiga M, et al. Young adult patients with short dissection length and thrombosed false lumen without ulcer-like projections are liable to have false-negative results of $D$-dimer testing for acute aortic dissection based on a study of 113 cases. Circ J 2006; 70: 1598-601.

26. Suzuki T, Distante A, Zizza A, et al. Diagnosis of acute aortic dissection by D-dimer: the International Registry of Acute Aortic Dissection Substudy on Biomarkers (IRAD-Bio) experience. Circulation 2009; 119: 2702-7.

27. Hazui H, Fukumoto H, Negoro N, et al. Simple and useful tests for discriminating between acute aortic dissection of the ascending aorta and acute myocardial infarction in the emergency setting. Circ J 2005; 69: 677-82.

28. Sakamoto K, Yamamoto Y, Okamatsu H, Okabe M. D-dimer is helpful for differentiating acute aortic dissection and acute pulmonary embolism from acute myocardial infarction. Hellenic J Cardiol 2011; 52: 123-7.

29. Gromadzinski L, Targonski R, Januszko-Giergielewicz B, Ostrowski P, Pruszczyk P. The significance of mitral and tricuspid valve systolic lateral annular velocities in the diagnosis of acute pulmonary embolism in patients with chronic heart failure. Arch Med Sci 2014; 10: 39-46.

30. Kruskal JB, Commerford PJ, Franks JJ, Kirsch RE. Fibrin and fibrinogen-related antigens in patients with stable and unstable coronary artery disease. N Engl J Med 1987; 317: 1361-5.

31. Hoffmeister HM, Jur M, Wendel HP, Heller W, Seipel L, Alterations of coagulation and fibrinolytic and kallikrein-kinin systems in the acute and postacute phases in patients with unstable angina pectoris. Circulation 1995; 91: 2520-7.

32. Menown IB, Mathew TP, Gracey HM, et al. Prediction of recurrent events by D-dimer and inflammatory markers in patients with normal cardiac troponin I (PREDICT) Study. Am Heart J 2003; 145: 986-92.
33. Oldgren J, Linder R, Grip L, Siegbahn A, Wallentin L. Coagulation activity and clinical outcome in unstable coronary artery disease. Arterioscler Thromb Vasc Biol 2001; 21: 1059-64. 